\title{
TMS Foundation Recognizes 2012 Supporters
}

The TMS Foundation thanks the following members and friends for their generous support in 2012. These gifts are critical to the success of current and future TMS Foundation programs, including scholarships, assistance and support of early career materials scientists and engineers in attaining their fullest potential, support of initiatives that have a significant impact on materials education, and advancement of the role of materials solutions in improving the quality of life in society and ensuring a sustainable environment.

To learn more about the TMS Foundation, visit http://www.tms.org /Foundation/tfhome.aspx.

(As of December 31, 2012)

PATRON (\$10-\$99)

Iver E. Anderson

Robert Bakish

Howard Bardwell

Leonid Beitelman

Claude Belleau

Daniel N. Beshers

Jose Juarez Borges-Filho

Brad L. Boyce

Stephan Broek

Ronald C. Campbell

John H. Canterford

Debbie Charles

Shengfeng Cheng

Akira Chiba

James R. Ciulik

Peter C. Collins

Jack Crane

Bruce Davis

Steven L. Dedmon

William Thomas Denholm

Alain Dery

Ian W. Donaldson

Jaroslaw W. Drelich

Jan Eigenhuis

Alan T. English

Cesare Ferron

John E. Flinn

Joy H. Forsmark

George D. Fulford

David U. Furrer

Walter L. Gage

Charles Geenen

Anthony F. Giamei

Marco V. Ginatta

George T. Gray, III

Howard T. Green

John A.S. Green

Vernon Griffiths

Mustafa Guclu

Osami Haruyama

J. Brian Haworth

Hani Henein

Richard W. Hertzberg

Tomoyuki Homma
Wayne R. Hopkins

Mihaiela Isac

Rafik Jerroudi

Mahesh C. Jha

Hojong Kim

Heinrich F. Klefenz

Axel E. Kranzmann

Venu G. Krishnardula

Tatsuo Kumagai

Henry L. Kurtz

Charles L. Kusik

Alexander I. Landa

Bennett C. Larson

Pascal Lavoie

Bhaskar S. Majumdar

Jacques Malan

Stanislaw T. Mandziej

Alexander Manuov

Koichiro Mashio

Jan W. Matousek

Arthur J. Mcevily

Alexander McLean

Terry R. McNelley

Francis J. Minden

Patrick B. Morgan

Paolo Murgia

Korukonda Linga Murty

Newell H. Orr

David D. Pearson

Michael W. Perkins

Jacko Pijper

Norbert L. Piret

Kaliat T. Ramesh

Ramesh C. Rao

Lothar H. Reh

Charles E.C. Rense

Douglas J. Robinson

William L. Roes

Michael Roesner-Kuhn

Joseph M. Rua

Wynn S. Sanders

W.J. Schlitt

Juracy Silva-Filho

Yongho Sohn

Joel E. Stein
Dion J. Sunderland

John M. Tartaglia

Jomar T. Thonstad

Francis O. Ugbo

Kurt Van Oosterwyck

Paulo Von Kruger

Tadao Watanabe

Richard M. Waterstrat

Robert Wheeler

Patrick Woolsey

Charles Fred Yolton

AMBASSADOR (\$100-\$249)

Diran Apelian

Richard J. Arsenault

Harold R. Clark

Theresa Coetsee

George E. Dieter, Jr.

John P. Hirth

Stanley M. Howard

William D. Macdonald

Christina E. Meskers

Thomas W. Montemarano

David P. Pope

Ramana G. Reddy

Daniel S. Schwartz

Dirk E. Verhulst

David L. Weight

PROVIDER (\$250-\$499)

Vincent M. Eannace

Alois J. Franke

FOUNDER (\$1,000-\$2,499)

Carl M. Cady

Phillip J. Mackey

David Alan Shifler

Garry W. Warren

CORPORATE

Hydro Aluminium Rolled Products Wagstaff Inc.
Arthur P. Turner 